Monatsschrift f. Geburtshülfe u. Gynäkologie 1917;46:3-4

\title{
Lieber Freund!
}

Von A. Döderlein

Auch der Dritte im Bunde möchte Dir heute seine besonderen Glückwünsche an dieser Stelle darbringen, gehört er doch mit Freund Schauta auch zu jenen, die in den 80 er Jahren des vorigen Jahrhunderts Dich in Berlin als einen der ersten, erfolgreichsten und gewandtesten Öperateure bewundern konnten und die davon für ihre ganze Lebensarbeit unvergänglichen Gewinn gezogen haben. Damals war Deine Klinik ein Wallfahrtsort für Gynäko-logen aus aller Welt. Ware es heute noch wie einst, so würden sich niit uns um Dich Kollegen aus aller Herren Lander scharen, hast Du Dir es doch stets angelegen sein lassen , die Internationalität der Wissenschaft zu pflegen. Deine kraftvolle Persönlichkeit fehlte auf keiner unserer Versammlungen, Deine markigen Worte, in welchen Sprachen sie auch erklangen, fanden stets getragen von tiefgründigem Wissen überall und immer den weitesten Widerhall. Was Du der deutschen Gynäkologie geschaffen, bleibt ein dauernder Gewinn, der alien Stürmen der Zeiten trotzt und Bewunderung und Dankbarkeit sichert.

Mit dieser von Deinen Schülern und Fretinden gegebenen Festschrift, niedergelegt in der von Dir geschaffenen und bis zum heutigen Tage in stets unermüdlicher Schaffensfreudigkeit und hingebender Arbeitskraft mustergültig geleiteten ,,Monatsschrift”, möchten wir Dir in ihr ein unvergängliches Zeichen unserer Ver-ehrung darbringen und zugleich durch die Beigabe eines voll-ständigen, von Dir selbst verfaßten und deshalb unanfechtbaren Verzeichnisses Deiner eigenen Arbeiten Dein Lebenswerk vor Dir selbst erstehen lassen. Deine Freunde wissen, wie hoch Du das unschätzbare Gut historischer Forschung einschätzest; es war uns deshalb ein besonderes Anliegen, durch Angliederung der Arbeiten Deines Vaters an Deine eigenen einen Ausschnitt der gynäkologischen Geschichte hier niederzulegen, der zeugt, wie innig die Entwicklung unseres Faches in mehr als einem halben

$1 *$

4

Jahrhundert ununterbrochen mit Martinsoher Forsehung ver-knüpft ist. Zwei Arbeiten Deiner Söhne in dieser Festschrift geben im Verèin mit verdienstvollen früheren dieser jüngsten Generation die Gewähr, daß dieser Bund in glücklichster Weise fortgesetzt wird, ein Geburtstagsgeschenk ganz besonderer Art.

Möchte unserer ,,Monatsschrift” Deine unersetzliche Arbeits-kraft noch recht lange beschieden sein und möchte sich unsere Wissenschaft noch vieler Früchte Deines lebendigen Geistes erfreuen dürfen.

München, Ostern 1917.

Döderlein.

Hochverehrter Meister!

Ein widriges Geschick verbietet es mir leider, Ihnen diesmal persönlich meine und zugleich Ihrer Schüler herzlichste Glück-wünsche zu Ihrem 70. Jubelfeste auszusprechen.

Groß ist die Zahl von Ärzten, die Sie in der langen Zeit Ihrer klinischen Tätigkeit um sich geschart und die Sie zu tüchtigen Vertretern unseres Faches haben heranbilden können. Wenn 
heute nur wenige von ihnen persönlich ihre Glückwünsche aus-sprechen, so liegt dies an der ernsten Zeit, welche den größten Teil im Dienste des Vaterlandes fesselt. Sie alle aber denken sieher heut hierher in Dankbarkeit an ihren alten Lehrer und mit Freude darüber, daß es Ihnen vergönnt ist, Ihren 70. Geburtstag in Frische und Rüstigkeit zu feiern.

Sie, verehrter Meister, sind uns alle Zeit ein Vorbild treuster PflichterfüIIung, rastlosen wissenschaftlichen Strebens und nie versagender Bereitwilligkeit gewesen, Ihren kranken Mitmenschen zu helfen, und daß wir das von Ihnen gelernt haben, das danken wir Ihnen heut nochmals von ganzem Herzen. Sie haben uns ge-lehrt, nicht die Krankheit, sondern den kranken Menschen zu behandeln.

Aber nicht nur für die rein praktische, sondern auch für die wissenschaftliche Seite unseres Faches haben Sie es verstanden, unser Interesse stets rege und wach zu erhalten. Davon gibt Zeugnis nicht nur die große Reihe der Veröffentlichungen Ihrer Schüler, sondern vor allem auch die vorliegende stattliche Festschrift, die zeigt, daß die Lust an wissenschaftlicher Arbeit nicht mit der Assistentenzeit erloschen ist. Daß ich mich hieran diesmal nicht beteiligen konnte, bedaure ich auf das lebhafteste. 